\title{
Optimal Design and Force Control of a Nine-Cable-Driven Parallel Mechanism for Lunar Takeoff Simulation
}

Wangmin $\mathrm{Yi}^{1,2,3^{*}}$, Yu Zheng ${ }^{2,4}$, Weifang Wang ${ }^{5}$, Xiaoqiang Tang ${ }^{1}$, Xinjun Liu ${ }^{1}$ and Fanwei Meng ${ }^{2,3}$

\begin{abstract}
Traditional simulation methods are unable to meet the requirements of lunar takeoff simulations, such as high force output precision, low cost, and repeated use. Considering that cable-driven parallel mechanisms have the advantages of high payload to weight ratio, potentially large workspace, and high-speed motion, these mechanisms have the potential to be used for lunar takeoff simulations. Thus, this paper presents a parallel mechanism driven by nine cables. The purpose of this study is to optimize the dimensions of the cable-driven parallel mechanism to meet dynamic workspace requirements under cable tension constraints. The dynamic workspace requirements are derived from the kinematical function requests of the lunar takeoff simulation equipment. Experimental design and response surface methods are adopted for building the surrogate mathematical model linking the optimal variables and the optimization indices. A set of dimensional parameters are determined by analyzing the surrogate mathematical model. The volume of the dynamic workspace increased by $46 \%$ after optimization. Besides, a force control method is proposed for calculating output vector and sinusoidal forces. A force control loop is introduced into the traditional position control loop to adjust the cable force precisely, while controlling the cable length. The effectiveness of the proposed control method is verified through experiments. A 5\% vector output accuracy and $12 \mathrm{~Hz}$ undulation force output can be realized. This paper proposes a cable-driven parallel mechanism which can be used for lunar takeoff simulation.
\end{abstract}

Keywords: Force control, Lunar takeoff simulation, Parallel robots, Surrogate mathematical model

\section{Introduction}

In the third phase of the Chinese lunar exploration project, the verification of lunar takeoff technology will be completed by Chang'e V probe [1]. During the initial phase of takeoff, because sufficient initial values of inertial measurements cannot be obtained, the guidance, navigation, and control system does not function in controlling the reentry capsule. Thus, the successful launch of the reentry capsule depends heavily on the launcher design, which should be verified by ground experiments. Therefore, a ground experimental device needs to be set up to precisely simulate the low-gravity environment on

\footnotetext{
*Correspondence: yiwangmin79@hotmail.com

1 Department of Mechanical Engineering, Tsinghua University, Beijing 100084, China

Full list of author information is available at the end of the article
}

the lunar surface, the thrust of the engine, and the disturbing force.

Currently, the available methods for simulating the low-gravity environment on the lunar surface could be classified into four categories, namely, the inertia compensation method [2], the buoyancy balance method [3], the rigidity parallel mechanism simulation method [4], and the sling suspension method [5]. The inertia compensation method is limited by short simulation time and high cost. The buoyancy balance method can establish a broad range of stable low-gravity environments, but the dynamic performances of the test objects are severely affected by water. The workspace of the rigid parallel mechanism is too small, while the sling suspension method can only support force along one direction. Considering that cable-driven parallel mechanisms (CDPMs) have the advantages of high payload to weight ratio, 
potentially large workspace, and high-speed motion [6], a parallel mechanism driven by nine cables is proposed for lunar takeoff simulation.

To perform lunar takeoff simulation experiments, a CDPM should have enough workspace to output the desired resultant force with high precision. The workspace of the CDPM is surrounded by a convex hull that is made up of fixed points [7]. The objectives of this study are to optimize the dimensions of the CDPM to meet the workspace requirements, and to design a control method to meet the force accuracy requirements.

So far, substantial progress has been made in the design of CDPMs. In Ref. [8], Ming pointed out that a cable system with $j$ end-effector DOFs requires at least $i=j+1$ cables, since the cable can only be in tension. Verhoeven [9] studied the feasible workspace considering pull and stiffness conditions. Gosselin focused on the dynamic workspace in solving the dynamic trajectory planning problem [10]. Merlet [11] proposed an algorithm to determine a polygonal approximation of the workspace border induced by a specific constraint. Previous studies on dimensional optimization design were based on workspace requirements, and the constraint condition was derived from tension and stiffness requirements [1214], which will greatly influence the accuracy of CDPMs. Tang et al. [15] proposed stiffness and tension may be considered as a whole to evaluate the workspace quality. Tension distribution is another issue in the study of CDPM. Researchers have taken advantage of the convex method [16], tension-level index [17], and gradient projection [18] to distribute tension.

Unlike previous studies, for the CDPM proposed in this paper, the distribution of cables is relatively concentrated, which means that the interactions among design variables can not be neglected. The response surface method (RSM) [19-21] is a multiparameter optimization method that is based on the concept of building a surrogate mathematical model to quantitatively describe the interactions among the design variables, and the relationship between the design variables and the performance index. To obtain a more accurate surrogate mathematical model with fewer test points, the experimental design method (DOE) is used to lay out the location of the sample points [21]. Thus, the first contribution of this study is the proposal of a novel optimization procedure based on the DOE and RSM to drive the structure of the CDPM for a given task.

The most frequently used control strategy is to control the cable length (or the angle of motor): a feedback control in the cable length is used for coordination to realize the desired cable length corresponding to a desired position of an end-effector. Motion convergence, using PD feedback control in the cable length coordinates, was proven with a Lyapunov function and Vector Closure by Kawamura et al. [22]. Fang et al. [23] proposed nonlinear feedback control laws in cable length coordinates, and the optimal tension distribution was considered to the advantage of the control laws. Werner et al. established an analogous model of the CDPM, then identified system parameters, pulley friction, and load, and finally implemented feed-forward and integral controllers to improve control accuracy [24, 25]. Other reported algorithms used in the control of CDPMs are composite control [26], computed torque control [27], robust PID control [28], and adaptive control [29]. However, previous studies could not meet the tension accuracy requirements for lunar takeoff simulation. In this study, a force loop is introduced into the traditional position control loop, and the force loop is used for the precise adjustment of tension.

The remainder of this paper is organized into six sections. In Section 2, we give the function requirements and a hardware overview of the CDPM. A kinematic model of the CDPM and an iterative program to calculate its workspace are given in Section 3. In Section 4, a surrogate mathematical model linking the structure parameters and the dynamic workspace is built based on the DOE and RSM. A set of optimized dimensional parameters can be obtained by analyzing the surrogate mathematical model. In Section 5, a hybrid force and position control method is proposed to improve the force accuracy. The prototype and experimental results of the proposed control method are presented in Section 6. Finally, conclusions and an outlook are given in Section 7.

\section{Task Analysis and System Overview}

Given the quality of the reentry capsule, the gravity data on the lunar surface, and the thrust data on the thruster, we can obtain the acceleration, velocity, and other parameters of the reentry capsule during the initial phase of takeoff. The maximum vertical acceleration is $2.314 \mathrm{~m} / \mathrm{s}^{2}$, and the maximum horizontal acceleration is $1.021 \mathrm{~m} / \mathrm{s}^{2}$. Therefore, the CDPM should be able to output the desired resultant force.

The index of the CDPM is derived from the scaled index of the real takeoff progress. The scaling ratios we adopted are listed as follows:

(1) The scaling ratio of acceleration is 1:1.

(2) The scaling ratio of the workspace is 1:4.

(3) The scaling ratio of quality should be the cube of the scaling ratio of the workspace. Therefore, the scaling ratio of quality is set as 1:64.

(4) From the equation $h=0.5 a t^{2}$, the square of the effective working time is proportional to the size of the workspace. Then, the scaling ratio of the effec- 
tive working time is $1: 2$, and the effective working time of the CDPM is set as one second.

According to the abovementioned requirements, the function requests of the CDPM are listed next. Considering the accuracy and real-time requirements, some indexes are added to a certain margin.

(1) The maximum load is $20 \mathrm{~kg}$.

(2) The maximum vertical acceleration is $2.4 \mathrm{~m} / \mathrm{s}^{2}$, and the maximum horizontal acceleration is $1.1 \mathrm{~m} / \mathrm{s}^{2}$.

(3) The error of the resultant force is less than $5 \%$.

(4) The moving platform should have six degrees of freedom.

Based on the abovementioned requirements, the CDPM should output the force with high precision and have enough dynamic workspace to output the desired acceleration for the desired time. The maximum quality, the maximum desired vertical acceleration, and the maximum desired horizontal acceleration of the moving platform are set as $20 \mathrm{~kg}, 2.4 \mathrm{~m} / \mathrm{s}^{2}$, and $\pm 1.1 \mathrm{~m} /$ $\mathrm{s}^{2}$ respectively. Integrating the acceleration and the velocity over time, and taking the safe distance of braking into consideration, the dynamic workspace should contain a cylindrical area of $\Phi 1.4 \mathrm{~m} \times 2.4 \mathrm{~m}$ and be as large as possible. In this study, we adopt the definition of dynamic workspace as presented in Ref. [30]. In order to achieve six-DOF motion control of the moving platform, at least seven cables are required. Redundant cables can optimize the dynamic workspace and attitude control ability of the CDPM, but more cables will increase the difficulty of controlling the mechanism precisely.

In this study, a nine-cable-driven parallel mechanism is introduced to perform the lunar takeoff simulation experiments. Figure 1 shows a schematic diagram of the nine-cable-driven parallel mechanism, in which the layout of connection points on the moving platform is "6-3" [31]. The lower three cables are directly connected to the winches, while the upper six cables go through pulleys connected to the winches on the bottom. The motion of the moving platform is realized by the upper six cables, and the lower 3 cables are used to exert the disturbing force.

The dimensional parameters can greatly affect the dynamic workspace. Thus, the "optimal design" in this study aims at obtaining a better set of dimension parameters for the CDPM to meet dynamic workspace requirements under cable tension constraints.

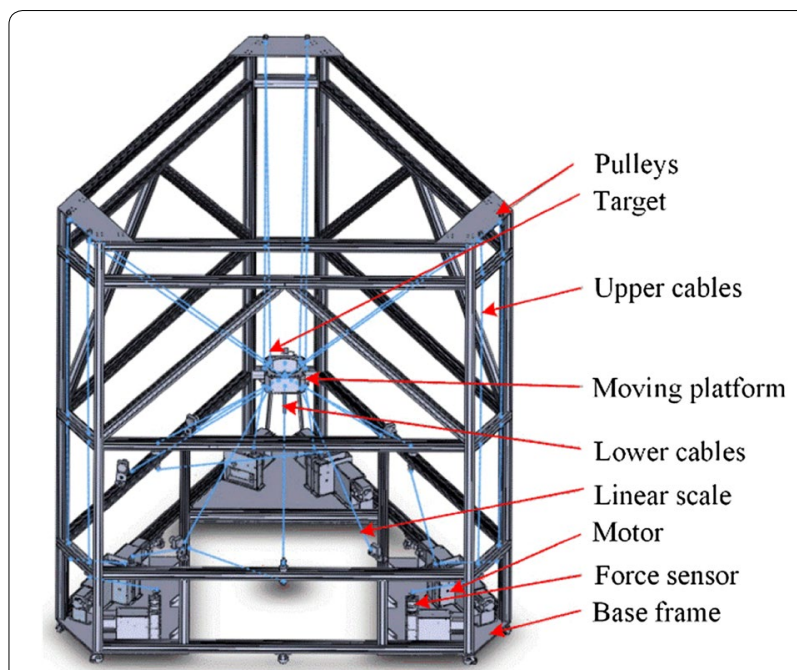

Figure 1 Schematic diagram of proposed nine-cable-driven parallel mechanism

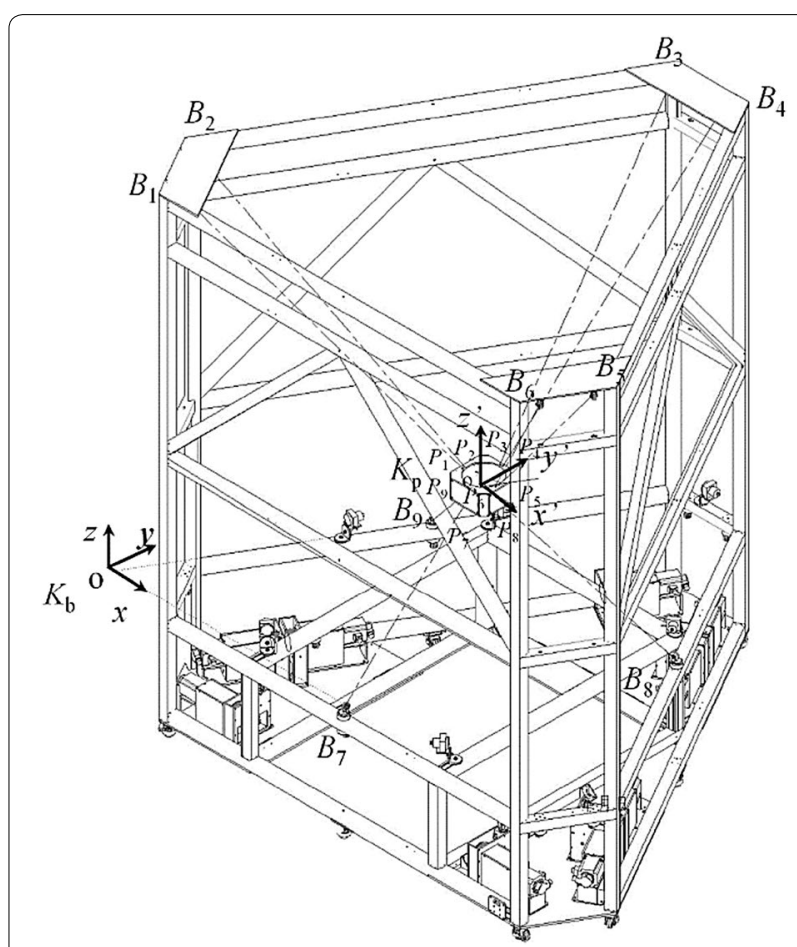

Figure 2 Mechanism model

\section{Kinematics and Workspace Calculation}

As shown in Figure 2, two coordinates are set up in the nine-cable-driven parallel mechanism: the global frame 
$K_{\mathrm{b}}$ is fixed at the base frame and the moving frame $\boldsymbol{K}_{\mathrm{p}}$ is connected to the moving platform. The symbols used in this paper are defined as follows: $B_{i}(i=1,2, \ldots, 9)$ represent the cable installation positions on the base frame, and $P_{i}(i=1,2, \ldots, 9)$ denote the cable installation positions on the moving platform. Vectors $\boldsymbol{p}_{i}(i=1,2, \ldots, 9)$ represent the vectors connecting point $o^{\prime}$ to points $P_{i}$, and $\boldsymbol{b}_{i}$ represent vectors connecting point $o$ to points $B_{i} \cdot \boldsymbol{f}_{\boldsymbol{i}}(i=1,2, \ldots, 9)$ are defined as the cable tension vectors along the $i$ cables. $\boldsymbol{f}_{\mathrm{p}}$ and $\boldsymbol{\tau}_{\mathrm{p}}$ represent external forces (except gravity) and torques acting on the platform. The position of the center of the end-effector in the fixed base frame $K_{\mathrm{b}}$ is defined as $\boldsymbol{o p}=(x, y, z)$, and the posture of the moving platform in $K_{\mathrm{b}}$ is defined as $(\Psi, \Phi, \gamma)$. Defining $\boldsymbol{R}$ as the transformation matrix from $\boldsymbol{K}_{\mathrm{p}}$ to $\boldsymbol{K}_{\mathrm{b}}$, the expression for $\boldsymbol{R}$ is given in Eq. (1):

$$
R=\left[\begin{array}{ccc}
c \boldsymbol{\Phi} c \gamma & -c \boldsymbol{\Phi} s \gamma & s \boldsymbol{\Phi} \\
s \boldsymbol{\Psi} \boldsymbol{s} \boldsymbol{\Phi} c \gamma+c \boldsymbol{\Psi} s \gamma & -s \boldsymbol{\Psi} \boldsymbol{s} \boldsymbol{\Phi} s \gamma+c \boldsymbol{\Psi} c \gamma & -s \boldsymbol{\Psi} c \boldsymbol{\Phi} \\
-c \boldsymbol{\Psi} \boldsymbol{s} \boldsymbol{\Phi} c \gamma+s \boldsymbol{\Psi} s \gamma & c \boldsymbol{\Psi} \boldsymbol{s} \boldsymbol{\Phi} s \gamma+s \boldsymbol{\Psi} s \gamma & c \boldsymbol{\Psi} c \boldsymbol{\Phi}
\end{array}\right]
$$

In Eq. (1), $s \Psi=\sin (\Psi), c \Psi=\cos (\Psi)$, and the remaining variables are as defined previously.

The unit vector along the $i$ th cable can be expressed as

$$
\boldsymbol{u}_{i}=\frac{\boldsymbol{l}_{i}}{\left|\boldsymbol{l}_{i}\right|}=\frac{\boldsymbol{b}_{i}-\boldsymbol{o p}-\boldsymbol{R} \cdot \boldsymbol{p}_{i}}{\left|\boldsymbol{b}_{i}-\boldsymbol{o p}-\boldsymbol{R} \cdot \boldsymbol{p}_{i}\right|}
$$

The force and torque equilibrium for the platform can be written as

$$
\boldsymbol{A}^{\mathrm{T}} \cdot \boldsymbol{t}+\boldsymbol{w}=0
$$

where $\boldsymbol{t}=\left[\begin{array}{llll}t_{1}, & t_{2}, & \ldots, & t_{9}\end{array}\right]^{\mathrm{T}}, \boldsymbol{w}=\left[\begin{array}{lll}\boldsymbol{f}_{\mathrm{p}}+m \boldsymbol{g}, & \boldsymbol{\tau}_{\mathrm{p}}\end{array}\right]^{\mathrm{T}}$, and $\boldsymbol{A}^{\mathrm{T}}=\left[\begin{array}{ccc}\boldsymbol{u}_{1} & \cdots & \boldsymbol{u}_{9} \\ \boldsymbol{p}_{1} \times \boldsymbol{u}_{1} & \cdots & \boldsymbol{p}_{9} \times \boldsymbol{u}_{9}\end{array}\right]$.

Considering the acceleration requirements of the moving platform, the expression for $\boldsymbol{f}_{\mathrm{p}}$ is given in Eq. (4):

$$
\boldsymbol{f}_{\mathrm{p}}=\left[-m \boldsymbol{a}_{1}-m \boldsymbol{a}_{2}-m \boldsymbol{a}_{3}\right]^{\mathrm{T}} \text {. }
$$

According to the parameters set in Section 2, $a_{1}= \pm 1.1 \mathrm{~m} / \mathrm{s}^{2}, a_{2}= \pm 1.1 \mathrm{~m} / \mathrm{s}^{2}, m=20 \mathrm{~kg}, a_{3}=2.4 \mathrm{~m} / \mathrm{s}^{2}$, and $\boldsymbol{\tau}_{\mathrm{p}}=[0,0,0]^{\mathrm{T}} \mathrm{N} \cdot \mathrm{m}$.

To study the dynamic workspace of the CDPM, Eq. (4) must be solved. A pose is part of the dynamic workspace only if a tension distribution result $t$ exists, and $\boldsymbol{t}_{\max } \geq \boldsymbol{t} \geq \boldsymbol{t}_{\min }>0$, where $\boldsymbol{t}_{\max }$ is the maximum tension and $\boldsymbol{t}_{\text {min }}$ is the minimum tension. The method first proposed by Lafourcade [32] is adopted in this study to solve Eq. (4), and the solution is shown in Eq. (5):

$$
\boldsymbol{t}=-\left(\boldsymbol{A}^{\mathrm{T}}\right)^{+} \cdot \boldsymbol{w}+\left(\boldsymbol{I}_{m \times m}-\left(\boldsymbol{A}^{\mathrm{T}}\right)^{+} \boldsymbol{A}^{\mathrm{T}}\right) \cdot \boldsymbol{t}_{\mathrm{d}},
$$

where $\boldsymbol{t}_{\mathrm{d}}=\left[t_{\mathrm{d}} t_{\mathrm{d}} t_{\mathrm{d}} t_{\mathrm{d}} t_{\mathrm{d}} t_{\mathrm{d}} t_{\mathrm{d}} t_{\mathrm{d}} t_{\mathrm{d}}\right]^{\mathrm{T}},\left(\boldsymbol{A}^{\mathrm{T}}\right)^{+}$is the MP inverse of $\boldsymbol{A}^{\mathrm{T}}$. The value of each element in $\boldsymbol{t}_{\mathrm{d}}$ is identical because the nine-cable-driven parallel mechanism is designed with modular parts.

Therefore, the dynamic workspace of the nine-cabledriven parallel mechanism can be determined using the following algorithm based on the Monte-Carlo method:

(1) The convex set of the frame is divided uniformly into 64000 parts. The center of each part is considered as a sample point.

(2) Choose a sample point.

(3) Calculate structure matrix $\boldsymbol{A}^{\mathrm{T}}$.

(4) Choose a desired tension $\boldsymbol{t}_{\mathrm{d}}$.

(5) $\boldsymbol{t}=-\left(\boldsymbol{A}^{\mathrm{T}}\right)^{+} \cdot \boldsymbol{w}+\left(\boldsymbol{I}_{m \times m}-\left(\boldsymbol{A}^{\mathrm{T}}\right)^{+} \boldsymbol{A}^{\mathrm{T}}\right) \cdot \boldsymbol{t}_{\mathrm{d}}$

(6) If $\boldsymbol{t}_{\max } \geq \boldsymbol{t} \geq \boldsymbol{t}_{\min }>0$, the chosen sample point belongs to the dynamic workspace. Otherwise, ignore the chosen sample point.

(7) Repeat (2)-(5) until all sample points are calculated.

\section{Optimal Design}

Most of the previous studies used a single-factor method to optimize the structure of the CDPM. For the CDPM proposed in this paper, the distribution of cables is relatively concentrated. Thus, the interactions among the design variables, which cannot be investigated with a single-factor method, would influence the dynamic workspace. To overcome the abovementioned shortcoming, we used the DOE to select experimental points and the RSM to build a surrogate mathematical model linking the structure variables and the performance indices. Then, by analyzing the surrogate mathematical model, the optimized variables were obtained while considering the interactions among the variables.

In this paper, we define the variable SUM_DWS as the number of sample points belonging to the dynamic workspace. The positions $B_{i}(i=1,2, \ldots, 9)$ are distributed around a circle with a radius of $2 \mathrm{~m}$, the height of the moving platform is $0.75 \mathrm{~m}$, and the height of the whole system is $3.5 \mathrm{~m} . B_{i}$ are $y$ axial symmetry in $\boldsymbol{K}_{\mathrm{b}}$, and $P_{\boldsymbol{i}}$ are $y$ axial symmetry in $\boldsymbol{K}_{\mathbf{p}}$. The circle formed by $B_{i}$ and that formed by $P_{i}$ are similar.

$\theta_{i}(i=1,2,3), R_{1}$, and $R_{2}$ define the optimal variables. These variables are shown in Figure 3, and the range of values for each variable is given in Table 1.

The Latin-hypercube method [33] was used to select the experimental points. This method is based on the principle of random probability orthogonal distribution. Thus, a response surface model with high precision 


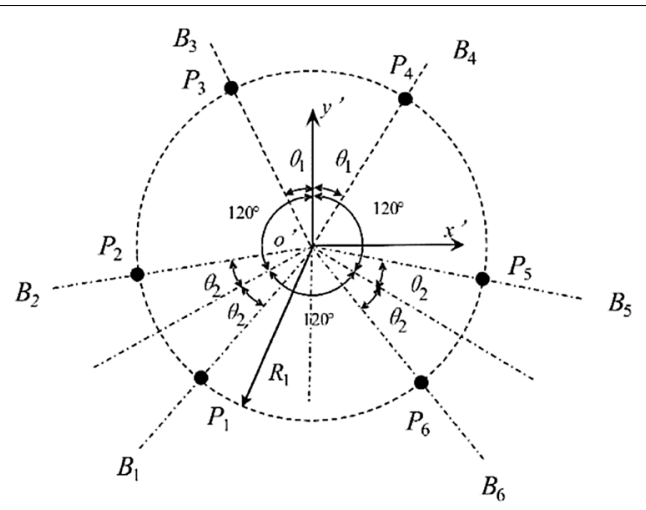

a Optimal variables on top surface of moving platform

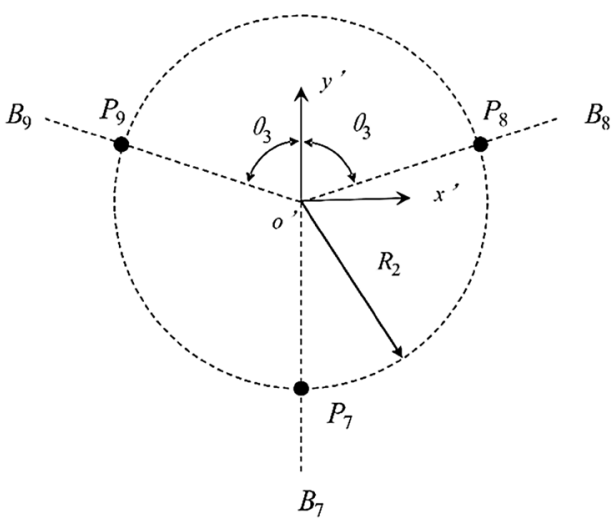

b Optimal variables on bottom surface of moving platform Figure 3 Optimal variables

Table 1 Variable names and levels

\begin{tabular}{lll}
\hline Variable name & Lower limit & Upper limit \\
\hline$\theta_{1}\left(^{\circ}\right)$ & 0 & 60 \\
$\theta_{2}\left(^{\circ}\right)$ & 0 & 60 \\
$\theta_{3}\left(^{\circ}\right)$ & 0 & 90 \\
$R_{1}(\mathrm{~m})$ & 0 & 0.5 \\
$R_{2}(\mathrm{~m})$ & 0 & 0.5 \\
\hline
\end{tabular}

can be obtained using fewer experimental points. The selected experimental points are shown in Figure 4.

A quadratic polynomial response surface model, as shown in Eq. (6), is used to establish the surrogate mathematical model linking the optimal variables and SUM_DWS.

$$
y(x)=\beta_{0}+\sum_{i=1}^{n} \beta_{i} x_{i}+\sum_{i=1}^{n} \beta_{i i} x_{i}^{2}+\sum_{i=1}^{n-1} \sum_{j=i+1}^{n} \beta_{i j} x_{i} x_{j} .
$$

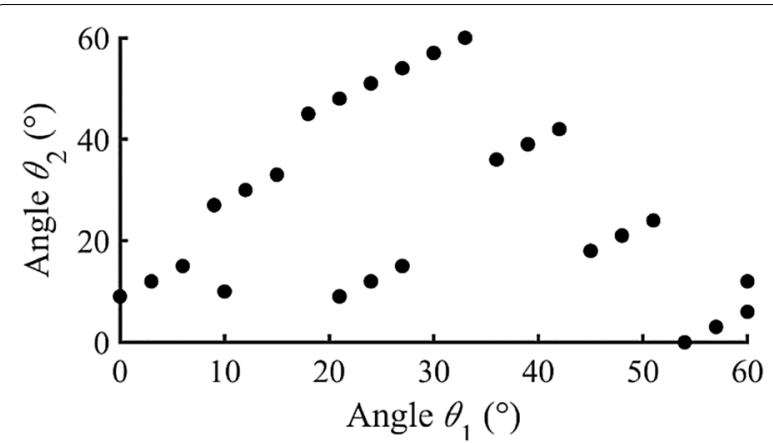

a Selected values of $\theta_{1}$ and $\theta_{2}$

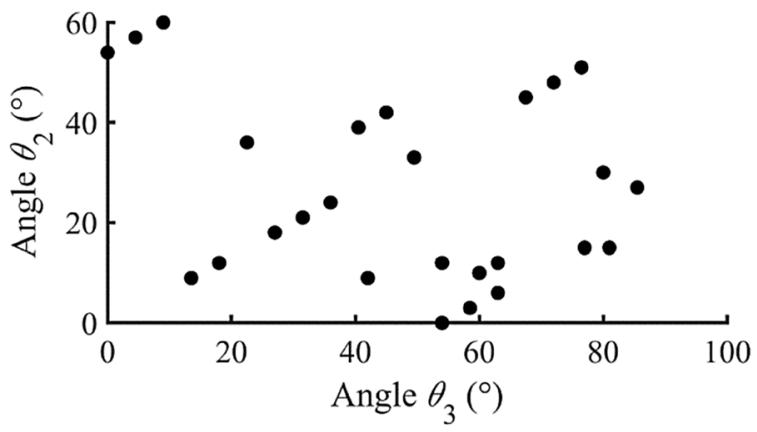

b Selected values of $\theta_{2}$ and $\theta_{3}$

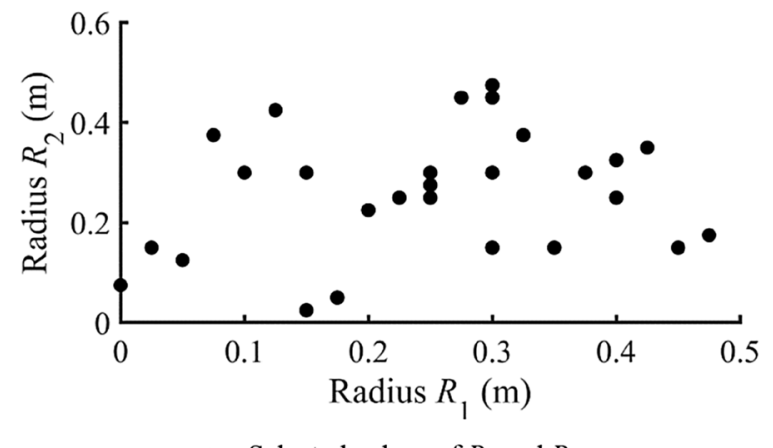

c Selected values of $R_{1}$ and $R_{2}$

Figure 4 Selected experimental points

In Eq. (6), $y(x)$ is the predictive value of the response surface model, $x_{i}$ is the $i$ th component of the $n$-dimensional independent variable. $\beta_{0}, \beta_{i}, \beta_{i i}$ and $\beta_{i j}$ are the coefficients of the polynomial, which are calculated by the least squares method. The surrogate mathematical model linking the optimal variables and the output response is given in Eq. (7):

$$
\begin{aligned}
\text { SUM_DWS } & =1534.18+1167.49 \theta_{1}+216.88 \theta_{2}+50.07 \theta_{3} \\
& -107236 R_{1}-34549.82 R_{2}-14.56 \theta_{1} \theta_{2} \\
& -6.39 \theta_{1} \theta_{3}-3980.62 \theta_{1} R_{1}-679.51 \theta_{1} R_{2} \\
& -0.19 \theta_{2} \theta_{3}+1370.92 \theta_{2} R_{1}+914.24 \theta_{2} R_{2} \\
& +879.23 \theta_{3} R_{1}+234.25 \theta_{3} R_{2}-68441.92 R_{1} R_{2} \\
& +7.67 \theta_{1}^{2}-5.32 \theta_{2}^{2}-0.97 \theta_{3}^{2}+309322 R_{1}^{2} \\
& -20260.13 R_{2}^{2} .
\end{aligned}
$$


Table 2 Calibration results

\begin{tabular}{lll}
\hline Optimal variable & $\begin{array}{l}\text { Determinant coefficients } \\
\boldsymbol{R}^{\mathbf{2}}\end{array}$ & $\begin{array}{l}\text { Multiple fitting } \\
\text { coefficient } \boldsymbol{R}_{\text {adj }}^{\mathbf{2}}\end{array}$ \\
\hline SUM_DWS & 0.9891 & 0.9617 \\
\hline
\end{tabular}

The determinant coefficient $R^{2}$ and multiple fitting coefficient $R_{\text {adj }}^{2}$ are used to verify the surrogate mathematical model, and the calibration results are shown in Table 2. Considering the whole response surface model as a hypersurface in a multidimensional space, we divide the response surface model into three 3-D response surface models, as shown in Figure 5. From Table 2, we can conclude that the response surface model meets the accuracy requirements. The mathematical relationship between the variables and the output can be approximated by a quadratic polynomial response surface.

Multiple sets of optimal solutions were obtained by searching for the maximum of the surrogate mathematical model. Then, the set of final optimized dimensional parameters were obtained while considering some restrictions on production, processing, and installations. Five groups of optimal solutions are shown in Table 3. The final optimized results are shown in the last line of Table 3.

After optimization, the dynamic workspace of the CDPM is shown in Figure 6. Figure 6 indicates that the dynamic workspace can meet the requirements. The comparison of variables before and after optimization are listed in Table 4, which indicates that the volume of the dynamic workspace increases by $46 \%$ after optimization. These optimized dimensional parameters will be applied in the construction of the CDPM.

\section{Hybrid Force and Position Control Method}

In this study, the CDPM was built to simulate the lowgravity environment on the lunar surface, the engine thrust, and the disturbing force. The gravity and the thrust can be considered as vector forces, while the disturbing force can be considered as a linear combination of sinusoidal forces at different frequencies. Thus, the CDPM should have the capacity to output a vector force and a sinusoidal force with high precision. Therefore, in this study, the resultant force of nine cables was set as the control target instead of the pose of the moving platform. Only the cable length can be controlled. The tension cannot be controlled precisely. The system could easily go out of control if only the tension is controlled. Therefore, this paper proposes a hybrid force and position control method that introduces a force feedback signal into the position control circuit of the cable drive unit. The force controller can adjust the tightness of the cable slightly,

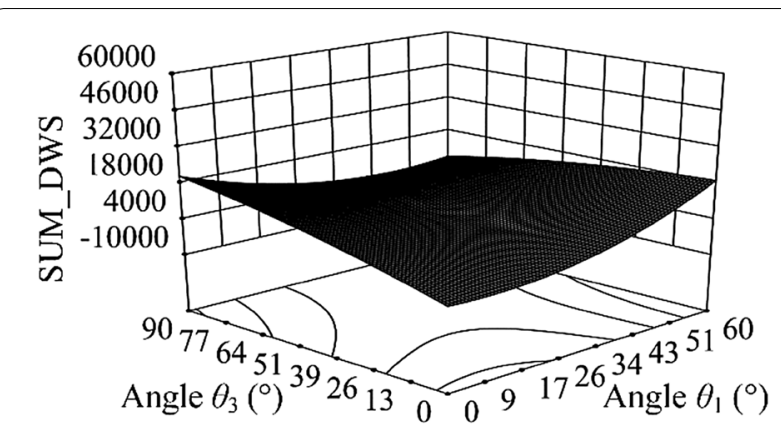

a Response surface model linking $\theta_{1}, \theta_{3}$, and SUM_DWS

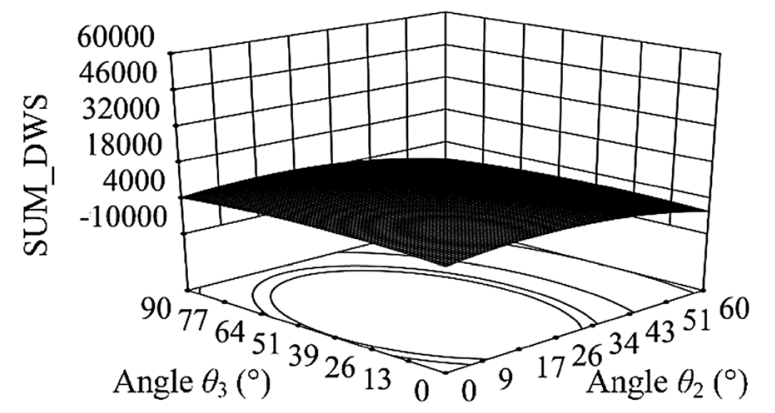

b Response surface model linking $\theta_{2}, \theta_{3}$, and SUM_DWS

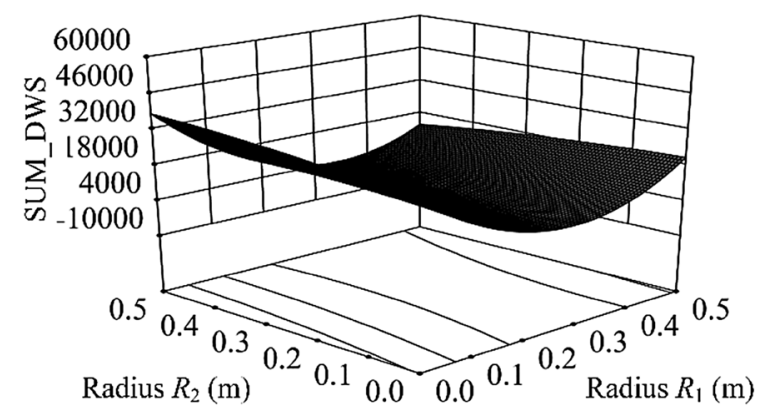

c Response surface model between $R_{1}, R_{2}$, and SUM_DWS

Figure 5 Response surface models

based on the cable force signals and the anticipated cable force, so as to control the cable force. The algorithm flow of the hybrid force and position control method is shown in Figure 7.

In each control cycle, the controller calculates the pose of the moving platform according to the pose sensor data. Then, the structure matrix $\boldsymbol{A}^{\mathrm{T}}$ is computed. According to the expected pose $\left(x_{\mathrm{d}} y_{\mathrm{d}} z_{\mathrm{d}} \Psi_{\mathrm{d}}, \Phi_{\mathrm{d}}, \gamma_{\mathrm{d}}\right)$ of the moving platform and the expected resultant force $\boldsymbol{w}_{\mathrm{d}}$, the expected cable length $\boldsymbol{L}_{\mathrm{d}}$ and the expected cable force $\boldsymbol{t}_{\mathrm{d}}$ are calculated. The force controller calculates the force control signal $\Delta \boldsymbol{l}$ according to the tension error $\boldsymbol{e}$. The cable drive unit overlays the force control signal $\Delta \boldsymbol{l}$, the expected 
Table 3 Optimal solutions

\begin{tabular}{lllllll}
\hline & Angle $\boldsymbol{\theta}_{\mathbf{1}}\left(^{(}\right)$ & Angle $\boldsymbol{\theta}_{\mathbf{2}}\left({ }^{\circ}\right)$ & Angle $\boldsymbol{\theta}_{\mathbf{3}}\left({ }^{\circ}\right)$ & Radius $\boldsymbol{R}_{\mathbf{1}}(\mathbf{m})$ & Radius $\boldsymbol{R}_{\mathbf{2}}(\mathbf{m})$ & SUM_DWS \\
\hline 1 & 3.25 & 6.62 & 57.56 & 0.26 & 0.05 & 8559.6 \\
2 & 4.73 & 3.61 & 77.06 & 0.25 & 0.11 & 7478.7 \\
3 & 5.85 & 6.17 & 62.59 & 0.23 & 0.08 & 7343.9 \\
4 & 4.82 & 8.03 & 53.24 & 0.28 & 0.11 & 7554 \\
5 & 2.69 & 4.76 & 54.77 & 0.29 & 0.11 & 8065.4 \\
6 & 5 & 5 & 60 & 0.25 & 0.1 & 6551 \\
\hline
\end{tabular}

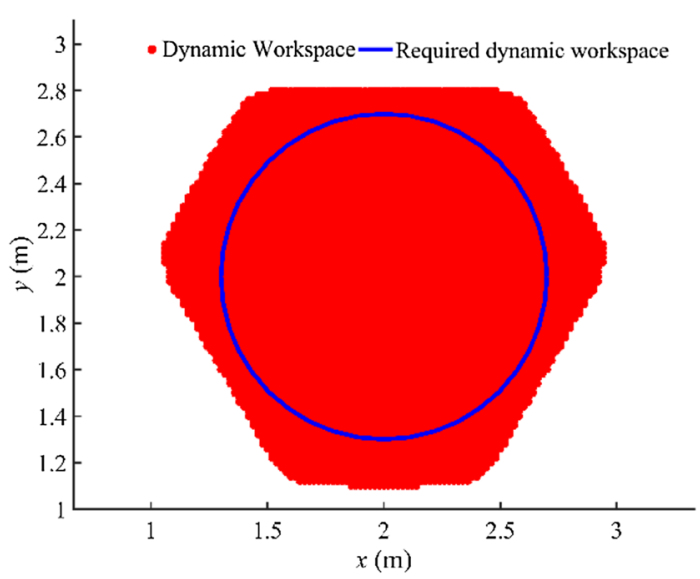

a Top view

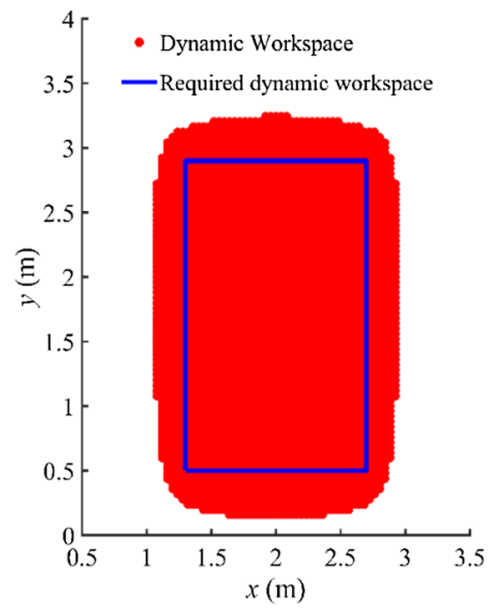

b Front view

Figure 6 Directional views of dynamic workspace length $\boldsymbol{L}_{\mathrm{d}}$ and the actual length $\boldsymbol{L}$, and completes the control of the cable length and cable force.

Six linear scales and six wire encoders are used to measure the pose of the moving platform, which can be calculated using the forward kinematics algorithm for the Stewart parallel mechanism. The layout of the linear scales and the wire encoders are shown in Figure 8, in which $S_{i}(i=1,2, \ldots, 6)$ represent the installation positions of the wire encoders, $L_{i}(i=1,2, \ldots, 6)$ represent the lengths of the linear scales, $M_{i}(i=1,2,3)$ represent the measurement points on the moving platform, and $m_{i}$ $(i=12,13,23)$ represent the lengths between the measurement points.

The forward kinematic model of the Stewart parallel mechanism is given in Eq. (8). Vectors $\boldsymbol{m}_{i}$ represent the vectors connecting point $o^{\prime \prime}$ to points $M_{i}$, and $s_{i}$ represent the vectors connecting point $o$ to point $S_{i}$. In Eq. (8), $\boldsymbol{R}$ and $\boldsymbol{o p}$ are as defined in Section 3:

$$
\left|\boldsymbol{L}_{i}\right|=\left|\boldsymbol{R} \boldsymbol{m}_{i}+\boldsymbol{o p}-\boldsymbol{s}_{i}\right|
$$

In Eq. (8), $\left|\boldsymbol{L}_{i}\right|, \boldsymbol{m}_{i}$, and $\boldsymbol{s}_{i}$ are known. The NewtonRaphson algorithm, which is shown in Eq. (9), is used to calculate the pose of the moving platform:

$$
x^{(k+1)}=x^{(k)}-F^{\prime}\left(x^{(k)}\right)^{-1} F\left(x^{(k)}\right),(k=0,1, \cdots) .
$$

The expected acceleration in each control cycle can be determined based on the expected resultant force $\boldsymbol{w}_{\mathrm{d}}$ and the load mass $\boldsymbol{m}$. By integrating the expected acceleration with time, the expected pose $\left(x_{\mathrm{d}} y_{\mathrm{d}} z_{\mathrm{d}} \Psi_{\mathrm{d}}, \Phi_{\mathrm{d}}, \gamma_{\mathrm{d}}\right)$ of the moving platform could be calculated. Defining $\boldsymbol{o} \boldsymbol{p}_{\mathrm{d}}=\left[x_{\mathrm{d}}, y_{\mathrm{d}}, z_{\mathrm{d}}\right]^{\mathrm{T}}, \Psi_{\mathrm{d}}, \Phi_{\mathrm{d}}$, and $\gamma_{\mathrm{d}}$ are used to calculate $\boldsymbol{R}_{\mathrm{d}}$. Then the expected length $\boldsymbol{L}_{\mathrm{d}}$ is obtained according

\begin{tabular}{|c|c|c|c|c|c|c|}
\hline & Angle $\theta_{1}\left({ }^{\circ}\right)$ & Angle $\theta_{2}\left({ }^{\circ}\right)$ & Angle $\theta_{3}\left({ }^{\circ}\right)$ & Radius $R_{1}(\mathrm{~m})$ & Radius $R_{2}(\mathrm{~m})$ & SUM_DWS \\
\hline Before optimization & 30 & 30 & 30 & 0.2 & 0.2 & 4397 \\
\hline After optimization & 5 & 5 & 60 & 0.25 & 0.1 & 6551 \\
\hline
\end{tabular}

Table 4 Comparison of variables before and after optimization 


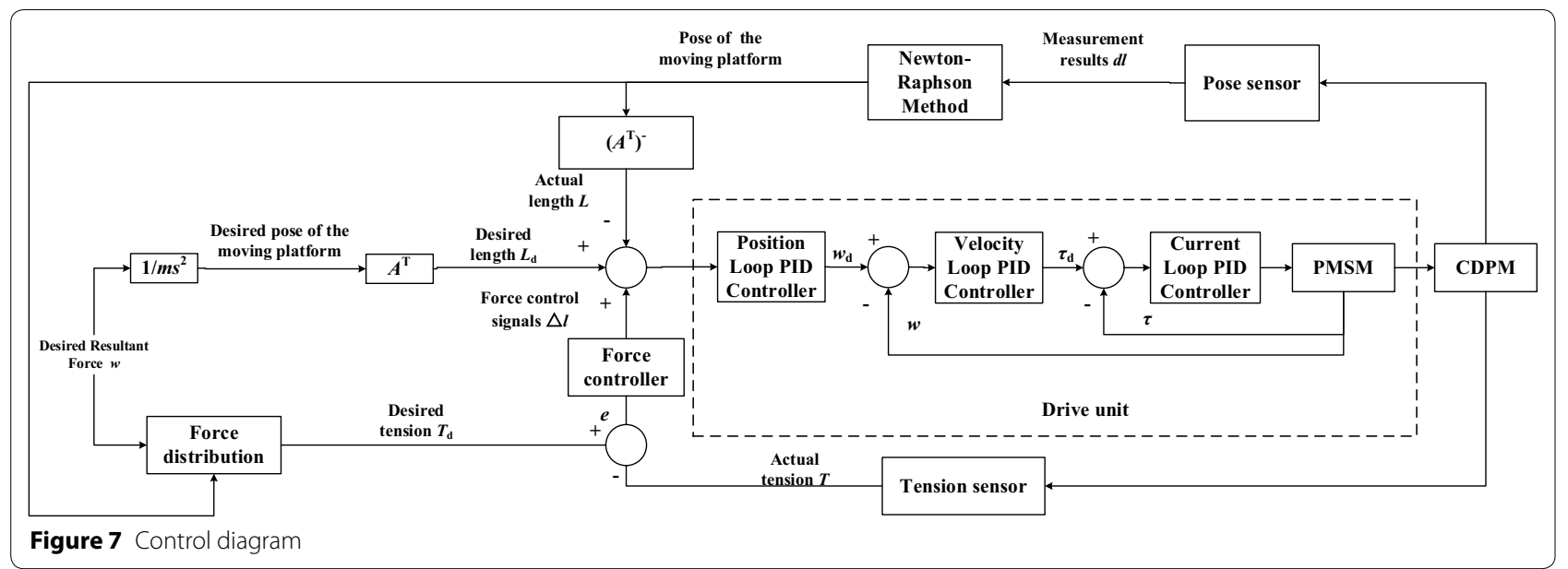

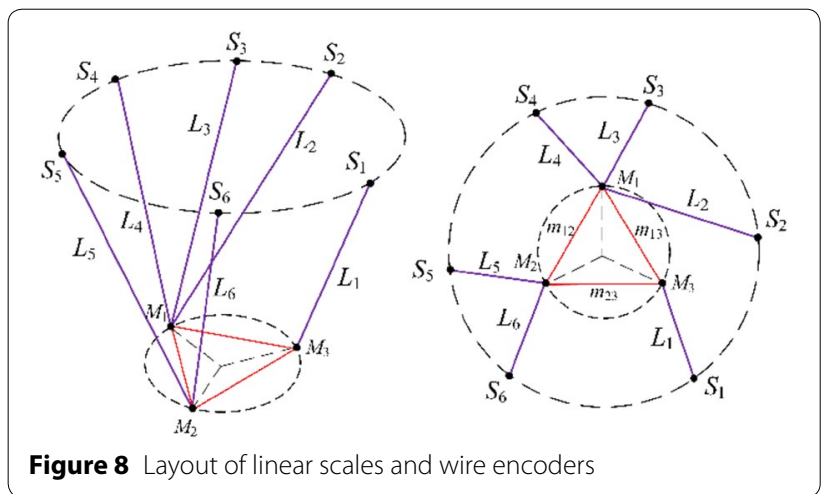

to Eq. (10). The permanent magnet synchronous motor (PMSM) in the drive unit drives the ropes to reach the desired lengths. Thus, the rough adjustment of tension is realized. The PMSM has a "current-speed-position" cascade control structure.

$$
\begin{aligned}
\boldsymbol{L}_{\mathrm{d}} & =\left[\begin{array}{lll}
\left|\boldsymbol{l}_{1 \mathrm{~d}}\right| & \left|\boldsymbol{l}_{2 \mathrm{~d}}\right| \cdots \mid & \left|\boldsymbol{l}_{9 \mathrm{~d}}\right|
\end{array}\right]^{\mathrm{T}}, \\
\left|\boldsymbol{l}_{i \mathrm{~d}}\right| & =\left|\boldsymbol{b}_{i}-\boldsymbol{o} \boldsymbol{p}_{\mathrm{d}}-\boldsymbol{R}_{\mathrm{d}} \cdot \boldsymbol{p}_{i}\right|,(i=1,2, \ldots, 9) .
\end{aligned}
$$

Meanwhile, according to the pose and the desired resultant force, the desired tension $\boldsymbol{t}_{\mathrm{d}}$ in each control cycle can be calculated. In the elastic range, the relationship between tension change $\mathrm{d} F_{i}$ and axial strain $\mathrm{d} l_{i} / l_{i}$ is given in Eq. (11):

$$
\mathrm{d} F_{i}=\frac{E S}{l_{i}} \mathrm{~d} l_{i} .
$$

For the CDPM examined in this study, the diameter of the cable is $0.5 \mathrm{~mm}$, and the modulus of elasticity is $206 \mathrm{GPa}$. Thus, if the tension changes by $5 \mathrm{~N}$, the rope deforms by $0.1 \mathrm{~mm}$. Therefore, control of the tension can be achieved by slightly controlling the deformation of the rope.

The force controller tightens or relaxes the rope based on the tension error $\boldsymbol{e}$, so that the expected tension is achieved. The control strategy is given in Eq. (12). The precise adjustment of tension is realized.

$$
\Delta l=\left\{\begin{array}{l}
0.05 \mathrm{~mm}, e<0,(\text { tighten rope }), \\
-0.05 \mathrm{~mm}, e>0, \text { (relax rope) }
\end{array}\right.
$$

The force distribution method is described next. The force distribution results are determined by Eq. (13):

$$
\boldsymbol{t}=\boldsymbol{t}_{\mathrm{up}}+\boldsymbol{t}_{\mathrm{down}}
$$

where,

$$
\begin{aligned}
\boldsymbol{t}_{\text {up }} & =\left[\begin{array}{llllll}
t_{1} & \cdots & t_{6} & 0 & 0 & 0
\end{array}\right]^{\mathrm{T}}, \\
\boldsymbol{t}_{\text {down }} & =\left[\begin{array}{llll}
0_{1 \times 6} & t_{7} & t_{8} & t_{9}
\end{array}\right]^{\mathrm{T}} .
\end{aligned}
$$

The desired tension of the lower cable should be set at a fixed value:

$$
\boldsymbol{t}_{\text {down }}^{\prime}=\left[\begin{array}{lll}
t_{7} & t_{8} & t_{9}
\end{array}\right]^{\mathrm{T}}=\left[\begin{array}{lll}
t_{\mathrm{pre}, 7} & t_{\mathrm{pre}, 8} & t_{\mathrm{pre}, 9}
\end{array}\right]^{\mathrm{T}} .
$$

Equation (16) is derived by substituting $\boldsymbol{t}_{\text {down }}$ into Eq. (3):

$$
J_{(6 \times 6)} \boldsymbol{t}_{\text {up }}^{\prime}=\boldsymbol{w}^{\prime} \text {. }
$$

After setting the tension of the lower cables as positive, the configuration of the nine-cable-driven parallel mechanism determines that the tension of upper cables is positive and the inverse matrix of $\boldsymbol{J}_{(6 \times 6)}$ exists. Thus, the force distribution results of upper cables are determined by Eq. (17):

$$
\boldsymbol{t}_{\text {up }}^{\prime}=\boldsymbol{J}_{(6 \times 6)}^{-1} w^{\prime}=\left[\begin{array}{lll}
t_{1} & \cdots & t_{6}
\end{array}\right]^{\mathrm{T}} .
$$

In the abovementioned force contribution method, the desired tension of the lower cables is set at fixed value to 
decrease the difficulty of control. Besides, the force distribution results can be adjusted by adjusting the preload value of the lower cables.

\section{Force Control Experiment}

\subsection{Prototype Design}

The prototype of the nine-cable-driven parallel mechanism is shown in Figure 9. The experimental platform consists of six parts: the basic frame, the drive unit, the force measurement module, the pose measurement module, the electrical control circuit, and the control software.

The aluminum alloy base frame is used to support and install the drive units, sensors, electrical circuits, and other hardware equipment.

The drive unit, shown in Figure 10, rotates the drum using the servo motor. A cable guiding device is included in the drive unit to ensure that the cable winding is not involved, and to reduce the friction force at the same time. The S-shaped cable guide channel and pulley are also used for cable guidance.

The $\mathrm{S}$ type tension sensor is selected for the experimental platform and is installed at the guidance channel. The tension sensor and its installation position are also shown in Figure 10. The accuracy class of the tension sensor is $0.3 \%$.

The pose measurement module includes six linear scales and six wire encoders with the Stewart configuration layout. The standard resolution of the wire encoder is $0.05 \mathrm{~mm}$ per pulse, and the accuracy of the wire encoder is $\pm 0.05 \% \mathrm{FS}$.

The core of the electrical control circuit, as shown in Figure 11, is the Turbo PMAC multi-axis controller from

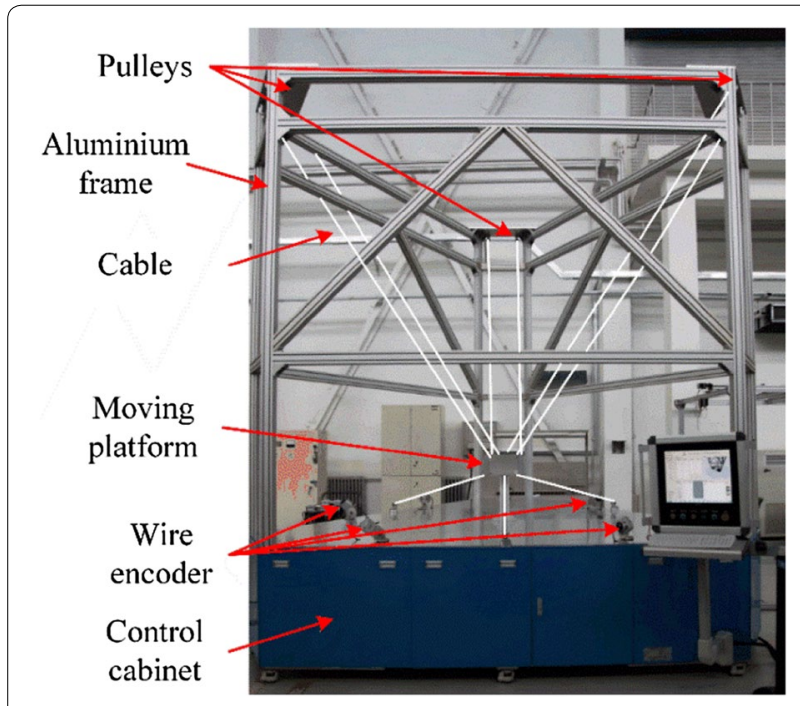

Figure 9 Nine-cable-driven parallel mechanism

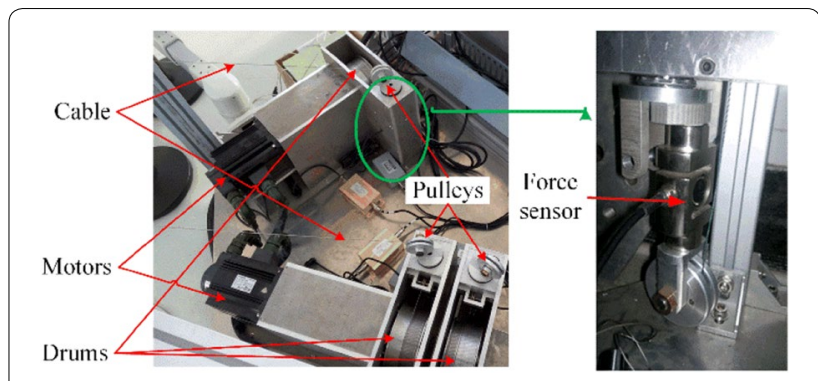

Figure 10 Drive unit and force sensor

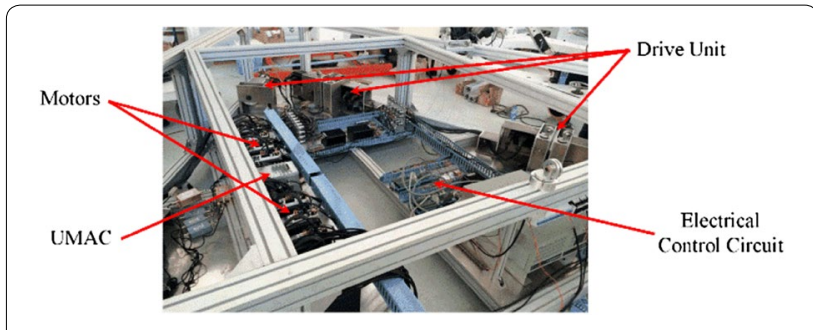

Figure 11 Electrical control circuit

Delta Tau Data Systems, Inc. The electrical circuit performs the functions of servo control, data acquisition of the force measurement and pose measurement modules, and load control.

\subsection{Experimental Results}

In this section, we briefly present the results of the force control experiments. First, the results of the constant vector force output experiments are presented. The experimental conditions were set as follows: the initial posture of the moving platform was $\left(0^{\circ}, 0^{\circ}, 0^{\circ}\right)$, the moving platform moved upwards vertically with an acceleration of $0.3 \mathrm{~m} / \mathrm{s}^{2}$, the quality of the moving platform was $20 \mathrm{~kg}$, the test time was $3 \mathrm{~s}$, and the control frequency was $500 \mathrm{~Hz}$.

Figure 12 shows the results of the constant vector force output experiments. Figure 12(a) indicates that the error of the joint force is less than $5 \%$. The desired and actual tensions are shown in Figure 12(b)-(d). The tensions of the upper six cables match with the expected values. The overall deviation in the tension of the upper six cables is caused by system errors, such as the deviation between the actual value and the theoretical value of the structure size, the quality of the load, and the moving platform. The tensions of the lower three cables fluctuate around the set values, and the fluctuation is less than $1.5 \mathrm{~N}$.

The results of the disturbing-force output experiments are given in Figure 13. In these experiments, the force/position hybrid control method is used to control the lower cable to output periodic wave forces at different frequencies. The desired tension is set to a constant 


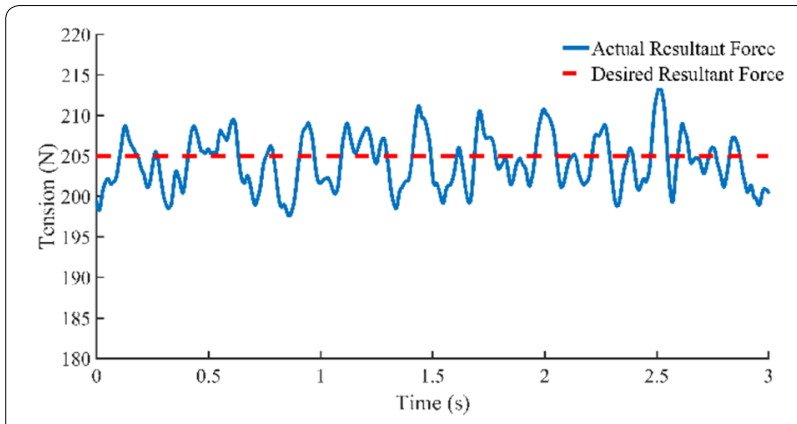

a Resultant force
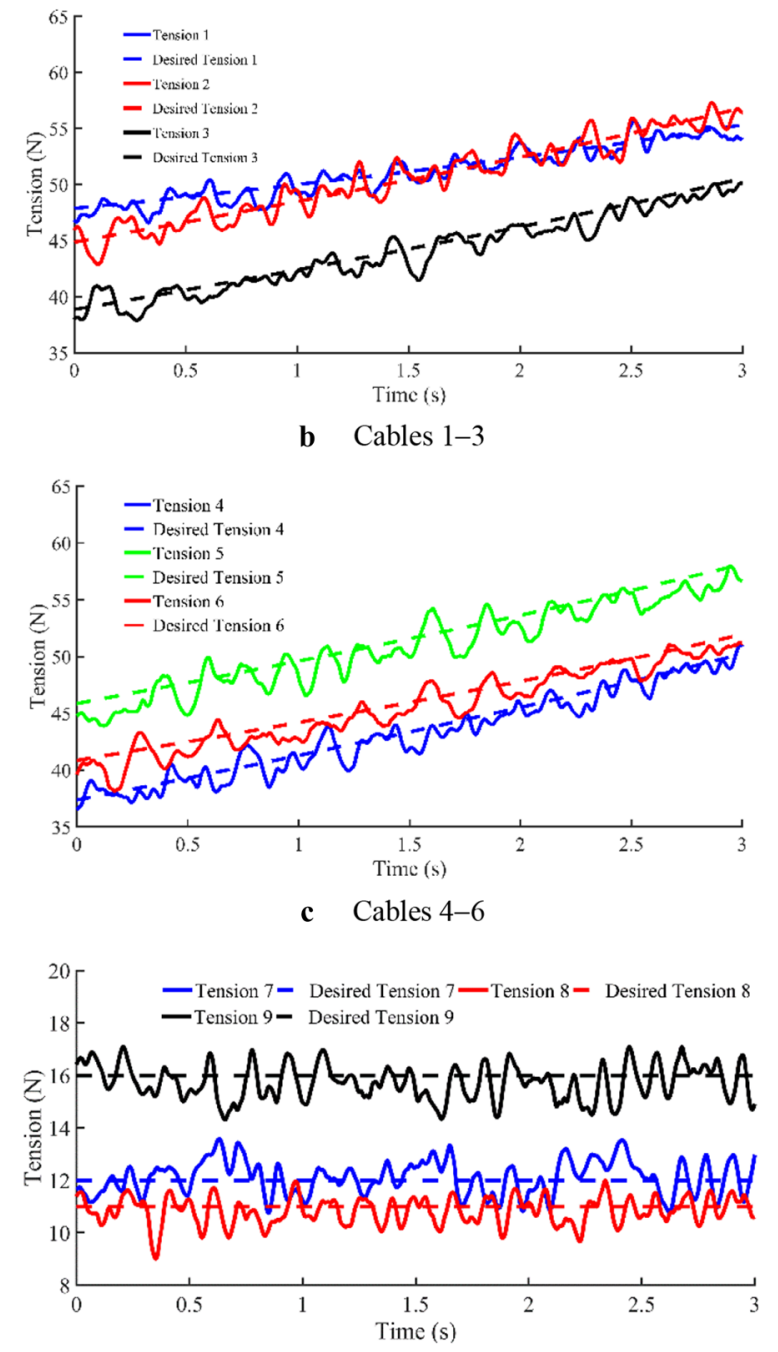

d Lower three cables

Figure 12 Results of constant vector force output experiments

force of $30 \mathrm{~N}$ with the periodic fluctuations, and the amplitude of the undulation force is $6 \mathrm{~N}$.

For wave forces of 2,3 , and $5 \mathrm{~Hz}$, the fluctuation of the actual output cable force is about $5.5,6$, and $7.5 \mathrm{~N}$, respectively. In the case of the $5 \mathrm{~Hz}$ wave force, the actual

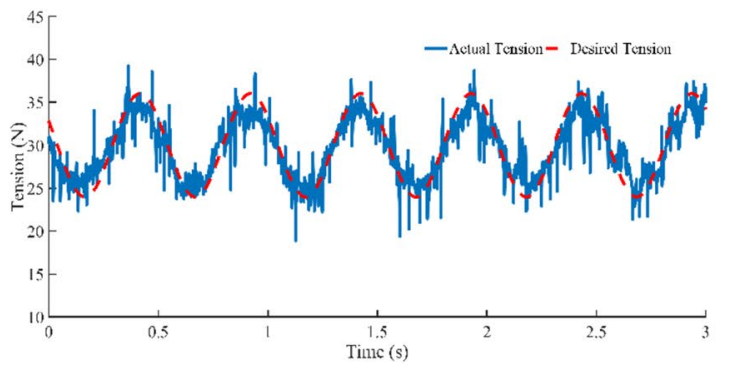

a $2 \mathrm{~Hz}$ wave force

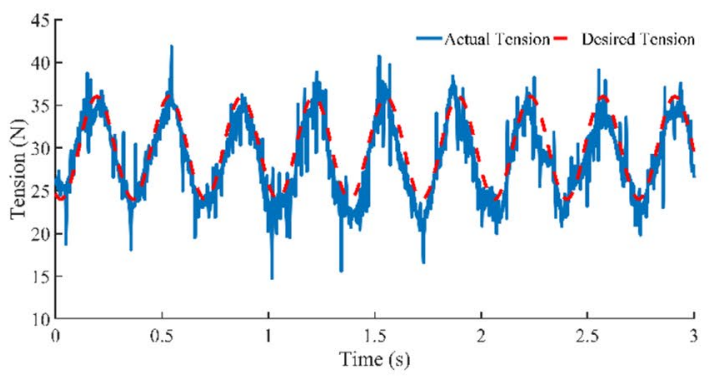

b $3 \mathrm{~Hz}$ wave force
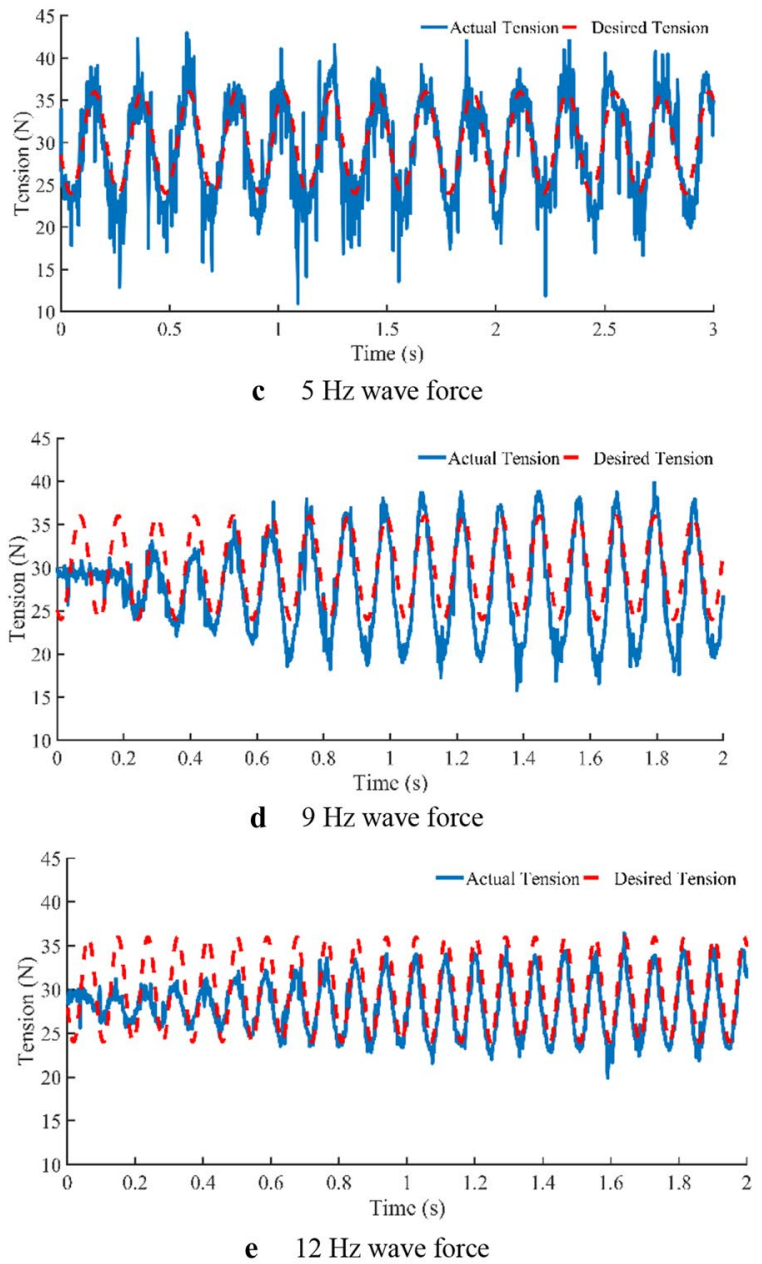

Figure 13 Results of undulation-force output experiments 
measurement glitch is larger, presumably because the inherent frequency of the cable-force-and-cable parallel mechanism is quite close to that of vibration coupling. For the wave force of $9 \mathrm{~Hz}$, the fluctuation of the actual output cable force is about $7.3 \mathrm{~N}$, and corresponding measurement glitch is relatively small. Similarly, for the wave force of $12 \mathrm{~Hz}$, the actual output fluctuation is about $4.5 \mathrm{~N}$, and both the cable-force average and the corresponding amplitude are less than the respective theoretical values.

The series of disturbing-force experimental results show that the hybrid force and position hybrid control method can control cable-force fluctuations within a certain range of frequencies. When the target cableforce fluctuation frequency is controlled from $5 \mathrm{~Hz}$ to $9 \mathrm{~Hz}$, the actual output cable-force fluctuation amplitude is slightly larger than that of the theoretical input. When the target cable-force frequency is less than $5 \mathrm{~Hz}$ or more than $9 \mathrm{~Hz}$, the actual output cable-force fluctuation amplitude is smaller than that of the theoretical input, and the overall target cable-force is reduced by approximately $1 \mathrm{~N}$.

\section{Conclusions}

(1) According to the requirements of lunar takeoff simulation experiments, the structure of the CDPM is presented using nine cables. The motion of the moving platform is achieved using the upper six cables, while the lower three cables are used to exert a disturbing force.

(2) For the nine-cable-driven parallel mechanism proposed in this paper, the mathematical relationship between the structure parameters and the dynamic workspace is described approximately by a quadratic polynomial response surface model. The nine-cable-driven parallel mechanism can cover a large dynamic workspace if the layout of the lower three cables is a regular triangle, the layout of the upper cables is similar to a triangle while the angle between the odd and even cables are $10^{\circ}-20^{\circ}$, the radius of the installation positions of the upper cable is $0.2-0.35 \mathrm{~m}$, and this radius is greater than that of the installation positions of the lower cables.

(3) A hybrid position and force control method is proposed for tension control. The experimental results indicate that, using this method, the cable-driven parallel mechanism can achieve 5\% accuracy in the vector output and an undulation-force output of $12 \mathrm{~Hz}$.

\section{Authors' contributions}

WY put forward the basic thought of optimal design and control method. YZ optimized the structure of CDPM, analyzed experimental results and wrote the manuscript. WW designed the tension control method and carried out experiments. XT and XL assisted with modeling, data analyzing and manuscript writing. FM designed experiments. All authors read and approved the final manuscript.

\section{Authors' Information}

Wangmin Yi, born in 1979, is currently a PhD candidate at Department of Mechanical Engineering, Tsinghua University, China, and the Executive Deputy Director of Beijing Engineering Research Center of the Intelligent Assembly Technology and Equipment for Aerospace Product, Beijing Institute of Space Environment Engineering, China. He received his master degree from Tsinghua University, China, in 2005. His research interests include robotic assembly technology, assembly equipment research and intelligent assembly robotic system based on compliance assembly.

Yu Zheng, born in 1993, is currently a PhD candidate at School of Mechanical Engineering and Automation, Beihang University, China. He received his master degree in Beijing Institute of Space Environment Engineering, China, in 2018.

Weifang Wang, born in 1989, is currently an engineer at Beijing Institute of Radio Measurement, Second Research Institute of the China Aerospace Science and Industry Group, China. He received his PhD degree from Tsinghua University, China, in 2016

Xiaoqiang Tang, born in 1973, is currently a professor at the Department of Mechanical Engineering, Tsinghua University, China. He received his PhD degree from Tsinghua University, China, in 2001.

Xinjun Liu, born in 1971, is currently a professor and the Director of Beijing Key Laboratory of Precision/Ultra-precision Manufacturing Equipments and Control, Tsinghua University, China, and the Assistant Director of the Department of Mechanical Engineering, Tsinghua University, China. He received his PhD degree from Yanshan University, China, in 1999.

Fanwei Meng, born in 1980, is currently an engineer at Beijing Institute of Space Environment Engineering, China Academy of Space Technology, China. He received his PhD degree from Tsinghua University, China, in 2012.

\section{Competing Interests}

The authors declare no competing financial interests.

\section{Funding}

Supported by National Natural Science Foundation of China (Grant No. 51405024).

\section{Author Details}

${ }^{1}$ Department of Mechanical Engineering, Tsinghua University, Beijing 100084, China. ${ }^{2}$ Beijing Institute of Spacecraft Environment Engineering, China Academy of Space Technology, Beijing 100094, China. ${ }^{3}$ Beijing Engineering Research Center of the Intelligent Assembly Technology and Equipment for Aerospace Product, Beijing Institute of Spacecraft Environment Engineering, Beijing 100094, China. ${ }^{4}$ School of Mechanical Engineering and Automation, Beihang University, Beijing 100191, China. ${ }^{5}$ Beijing Institute of Radio Measurement, Second Research Institute of the China Aerospace Science and Industry Group, Beijing 100854, China.

Received: 3 July 2018 Revised: 24 February 2019 Accepted: 7 August 2019

Published online: 29 August 2019

\section{References}

[1] P J Ye, J C Huang, Z Z Sun, et al. The process and experience in the development of Chinese lunar probe. Sci. Sin. Tech., 2014, 44(6): 543-558. (in Chinese)

[2] V Belser, J Breuninger, R Laufer, et al. Aerodynamic and engineering design of a 1.5 seconds high quality microgravity drop tower facility. Acta Astronautica, 2016, 129(1): 335-344. 
[3] J Zhao, N Liu, B Wu. Human motion simulation comparison research between neutral buoyancy and weightless environment. Manned Spaceflight, 2014, 20(6): 517-519. (in Chinese)

[4] PYang, HTWu, X L Yang, et al. Study of 6 DOF microgravity simulation platform. Machinery Design \& Manufacture, 2015, 3: 5-10. (in Chinese)

[5] L F Li, Z Q Deng, H B Gao, et al. Active gravity compensation test bed for a six-DOF free-flying robot. 2015 IEEE International Conference on Information and Automation, Lijiang, China, August 8-10, 2015: 3135-3140.

[6] X QTang. An overview of the development for cable-driven parallel manipulator. Advances in Mechanical Engineering, 2014(1): 1-9.

[7] W F Wang, X Q Tang, Z F Shao, et al. Design and analysis of a wire-driven parallel mechanism for low-gravity environment simulation. Advances in Mechanical Engineering, 2014, 52(9): 1-8.

[8] A Ming, T Higuchi. Study on multiple degree-of-freedom positioning mechanism using wires (part 1) - Concept, design and control. International Journal of the Japan Society for Precision Engineering, 1994, 28(2): 131-138.

[9] R Verhoeven. Analysis of the workspace of tendon-based Stewart platforms. University of Duisburg-Essen PhD: University of Duisburg-Essen Press, 2004.

[10] C Gosselin. Global planning of dynamically feasible trajectories for threeDOF spatial cable-suspended parallel robots. Cable-Driven Parallel Robots, Berlin: Springer, 2013: 3-22.

[11] J P Merlet. Computing cross-sections of the workspace of a cable-driven parallel robot with 6 sagging cables having limited lengths. Advances in Robot Kinematics, 2018, 8: 1-8

[12] R Yao, X Q Tang, J S Wang, et al. Dimensional optimization design of the four-cable-driven parallel manipulator in FAST. IEEE/ASME Transactions on Mechatronics, 2010, 15(6): 932-941.

[13] Y Bo, W W Shang. Wrench-feasible workspace based optimization of the fixed and moving platforms for cable-driven parallel manipulators. Robotics and Computer-Integrated Manufacturing, 2014, 30(6): 629-635.

[14] E Hernandez, S I Valdez, G Carbone, et al. Design optimization of a cable-driven parallel robot in upper arm training-rehabilitation processes. Multibody Mechatronic Systems, 2018, 54(3): 413-423.

[15] X Q Tang, L W Tang, J S Wang, et al. Workspace quality analysis and application for a completely restrained 3-Dof planar cable-driven parallel manipulator. Journal of Mechanical Science and Technology, 2013, 27(8): 2391-2399

[16] A Ghasemi, M Eghtesad, M Farid. Workspace analysis for planar and spatial redundant cable robots. American Control Conference, Seattle, Washington, USA, June 11-13, 2009: 2389-2394.

[17] W B Lim, S H Yeo, G L Yang. Optimization of tension distribution for cabledriven manipulators using tension-level index. IEEE/ASME Transactions on Mechatronics, 2014, 19(2): 676-683.

[18] W B Lim, S H Yeo, G L Yang, et al. Tension optimization for cable-driven parallel manipulators using gradient projection. IEEE/ASME International Conference on Advanced Intelligent Mechatronics, Budapest, Hungary, July 3-7, 2011: 73-78.

[19] CY Zhang, L K Song, CW Fei, et al. Advanced multiple response surface method of sensitivity analysis for turbine blisk reliability with multi-physics coupling. Chinese Journal of Aeronautics, 2016, 29(4): 962-971.

[20] Y Wang, S Jin, S Penugonda, et al. Variability analysis of crosstalk among differential vias using polynomial-chaos and response surface methods. IEEE Transaction on Electromagnetic and Compatibility, 2017, 59(4): 1368-1378.

[21] Y K Sui, H P Yu. Improvement of response surface methodology and its application to engineering optimization. Beijing: Science Press, 2011. (in Chinese)

[22] S Kawamura, H Kino, C Won. High-speed manipulation by using parallel wire-driven robots. Robotica, 2000, 18(1): 13-21.

[23] S Fang, D Franitza, M Torlo, et al. Motion control of a tendon-based parallel manipulator using optimal tension distribution. IEEE/ASME Transactions on Mechatronics, 2004, 9(3): 561-568.

[24] W Kraus, V Schmidt, P Rajendra, et al. System identification and cable force control for a cable-driven parallel robot with industrial servo drives. IEEE International Conference on Robotics \& Automation, Hong Kong, China, May 31-June 7, 2014: 5921-5926.

[25] W Kraus, M Kessler, A Pott. Pulley friction compensation for winchintegrated cable force measurement and verification on a cable-driven parallel robot. IEEE International Conference on Robotics \& Automation, Washington, USA, May 26-30, 2015: 1627-1632.

[26] M A Khosravi, H D Taghirad. Dynamic modeling and control of paralle robots with elastic cables: Singular perturbation approach. IEEE Transactions on Robotics, 2014, 30(3): 694-704.

[27] A Aflakiyan, H Bayani, M T Masouleh. Computed torque control of a cable suspended parallel robot. Proceedings of the 3rd RSI International Conference on Robotics and Mechatronics, Tehran, Iran, October 7-9, 2015: 749-754.

[28] M A Khosravi, H D Taghirad. Robust PID control of fully-constrained cable driven parallel robots. Mechatronics, 2014, 24(2): 87-97.

[29] R Babaghasabha, M A Khosravi, H D Taghirad. Adaptive control of kntu planar cable-driven parallel robot with uncertainties in dynamic and kinematic parameters. Cable-Driven Parallel Robots: Proceedings of the Second International Conference on Cable-Driven Parallel Robots. Berlin, Springer International Publishing, 2015: 145-159.

[30] G Barrette, C M Gosselin. Determination of the dynamic workspace of cable-driven planar parallel mechanisms. Journal of Mechanical Design, 2005, 127(2): 242-248.

[31] P H Borgstrom, B L Jordan, G S Sukhatme, et al. Rapid computation of optimally safe tension distributions for parallel cable-driven robots. IEEE Transactions on Robotics, 2009, 25(6): 1271-1281.

[32] P Lafourcade, M Libre, C Reboulet. Design of a parallel wire-driven manipulator for wind tunnels. Proceedings of the Workshop on Fundamental Issues and Future Research Directions for Parallel Mechanisms and Manipulators, Quebec City, Quebec, Canada, October 3-4, 2002: 187-194.

[33] Q S Xu, Y Yang, Y J Liu, et al. An improved Latin hypercube sampling method to enhance numerical stability considering the correlation of input variables. IEEE Access, 2017, 5: 15197-15205.

\section{Submit your manuscript to a SpringerOpen ${ }^{\circ}$ journal and benefit from:}

- Convenient online submission

- Rigorous peer review

- Open access: articles freely available online

- High visibility within the field

- Retaining the copyright to your article

Submit your next manuscript at springeropen.com 